\title{
Anti-inflammatory effect of hesperidin enhances chondrogenesis of human mesenchymal stem cells for cartilage tissue repair
}

Shipeng Xiao', Wenguang Liu', Jianqiang Bi ${ }^{2}$, Shenghou Liu', Heng Zhao ${ }^{1}$, Ningji Gong ${ }^{3}$, Deguo Xing ${ }^{4}$, Hongwei Gao ${ }^{4}$ and Mingzhi Gong ${ }^{4^{*}}$

\begin{abstract}
Background: Articular cartilage diseases are considered a major health problem, and tissue engineering using human mesenchymal stem cells (MSCs) have been shown as a promising solution for cartilage tissue repair. Hesperidin is a flavonoid extract from citrus fruits with anti-inflammatory properties. We aimed to investigate the effect of hesperidin on MSCs for cartilage tissue repair. MSCs were treated by hesperidin, and colony formation and proliferation assays were performed to evaluate self-renewal ability of MSCs. Alcian blue staining and Sox9 expression were measured to evaluate chondrogenesis of MSCs. Secretion of pro-inflammatory cytokines IFN- $\gamma$, IL-2, IL-4 and IL-10, and expression of nuclear factor kappa B (NF-KB) subunit p65 were also assessed.
\end{abstract}

Results: Hesperidin improved self-renewal ability and chondrogenesis of MSCs, inhibited secretion of pro-inflammatory cytokines IFN- $\gamma$, IL-2, IL-4 and IL-10, and suppressed the expression of p65. Overexpression of p65 was able to reverse the hesperidin inhibited secretions of pro-inflammatory cytokines, and abolish the enhancing effect of hesperidin on chondrogenesis of MSCS.

Conclusion: Hesperidin could serve as a therapeutic agent to effectively enhance chondrogenesis of human MSCs by inhibiting inflammation to facilitate cartilage tissue repair.

Keywords: Articular cartilage, Hesperidin, Mesenchymal stem cells, Chondrogenesis, Inflammation

\section{Background}

Highly specialized articular cartilage consists of chondrocytes that are embedded in a rich extracellular matrix, mostly constituted by collagen, proteoglycans, and water $[1,2]$. The cartilage provides a low friction on the articular surfaces of the bone, and serves as an impact absorber for joint movement by covering the load-bearing surface $[3,4]$. It possesses a low capacity for repair and regeneration due to the absence of vascular, lymphatic and neural networks as well as the lack of sufficient progenitor cells within the cartilage $[5,6]$. Hence, once articular cartilage is injured and damaged,

\footnotetext{
* Correspondence: gongmingzhi2008@sina.com

${ }^{4}$ Department of Trauma and Orthopedics, The Second Hospital of Shandong University, Jinan 250033, Shandong, China

Full list of author information is available at the end of the article
}

it cannot be spontaneously repaired. In trauma or more commonly in degenerative joint diseases like rheumatoid arthritis and osteoarthritis, which affect at least 15\% US population, structure and function of the articular cartilage tissues are often both impaired [7, 8]. Articular cartilage diseases undoubtedly bring a large financial burden to the individuals as well as the health system worldwide, and are therefore regarded as a foremost health problem particularly in developed countries $[7,9]$.

Tissue engineering provides an exciting alternative approach for treating articular cartilage diseases via the development of biological substitutes. Recent reports demonstrated that human stem cells, especially mesenchymal stem cells (MSCs), produced positive outcomes in the treatment of articular cartilage diseases [10]. Multiple approaches have been taken to utilize MSCs,

(c) The Author(s). 2018 Open Access This article is distributed under the terms of the Creative Commons Attribution 4.0 International License (http://creativecommons.org/licenses/by/4.0/), which permits unrestricted use, distribution, and 
with some efforts focusing on their in vitro expansion while others on in vitro differentiation. For instance, MSCs expanded in vitro were reported to be successfully transplanted into the defective articular cartilage of both animals and human patients, which underwent differentiation in vivo that eventually led to regeneration of osteochondral tissue [11]. In addition, human MSCs can be obtained from a variety of adult tissues, expanded with ease, and subsequently differentiated into matrix-producing chondrocytes in vitro $[12,13]$, eventually leading to the formation of hyaline articular cartilage. Due to the potential teratoma formation of pluripotent stem cells (e.g., induced pluripotent stem cells or embryonic stem cells), they are less preferable compared to MSCs in cartilage tissue engineering [14]. However, cartilage tissue engineering has yet to be proven effective in clinical use. The structural and functional properties of native articular cartilage have not been fully adopted by the tissue-engineered cartilage $[15,16]$. Therefore, to date, there is no reliable long-term therapeutic strategy for articular cartilage repair [17]. Conventional therapies (e.g., microfracture, mosaicplasty and $\mathrm{ACI}$ ) or traditional therapies (e.g., joint surgery) possess several shortcomings. In joint surgery, implantation of a prosthetic device is performed to replace the living cartilage tissue, which can marginally rescue joint functions but for merely $10-15$ years. This procedure also poses additional risks of post-surgery complications, including infection and inflammation [18]. Thus, an efficient treatment to successfully repair or regenerate articular cartilage tissues is urgently needed.

Hesperidin is a natural flavonoid that possesses anti-inflammatory properties in many disease models. For example, hesperidin has been shown in rodent model to reduce inflammation as well as inflammatory pain through suppression of cytokine production, NF- $\mathrm{kB}$ activity, and oxidative stress [19]. In a similar manner, in a mouse model of skin damage induced by ultraviolet B irradiation, hesperidin was demonstrated to inhibit oxidative stress and inflammation [20], by down-regulation of cytokine production including TNF- $\alpha$, IL-1 $\beta$, IL- 6 and IL-10 [21]. However, the effect of hesperidin on the immune responses during chondrogenesis of MSCs has not yet been reported.

In the current study, we hypothesized that hesperidin could enhance self-renewal and chondrogenesis of isolated human MSCs in vitro, which could then facilitate their in vitro expansion and differentiation at a large scale for clinical cartilage tissue repair.

\section{Results}

\section{Hesperidin improves self-renewal ability of MSCs}

The chemical structure of hesperidin was identified as shown in Fig. 1a. Here MSCs cells were challenged with different doses of hesperidin $(0,1,5$ and $10 \mu \mathrm{M})$, and the self-renewal capacity was assessed by colony formation and proliferation assays. Both the relative number and average size of colonies were significantly increased following hesperidin treatments up to $5 \mu \mathrm{M}$ (Fig. 1b, c). Similarly, the cell viability determined using CCK-8 method clearly demonstrated that hesperidin markedly stimulated cell proliferation (Fig. 1d). However, high dose of hesperidin $(10 \mu \mathrm{M}$ in our system) induced slight inhibition on both colony formation and cell proliferation (Fig. 1b, c, d). Thus, $5 \mu \mathrm{M}$ of hesperidin was chosen as the optimal dosage for the subsequent experiments in the current study, and to our best knowledge, these findings provided the first evidence that hesperidin improved self-renewal ability of patient-derived MSCs.

\section{Hesperidin enhances chondrogenesis of MSCs}

Next, we sought to evaluate the possible effects of hesperidin on chondrogenesis potential of MSCs. Chondrogenesis was induced in the MSCs for 14 days upon hesperidin treatment. As shown in Fig. 2a, Alcian Blue staining showed significant increase of chondrogenesis in hesperidin-treated MSCs. These phenotypic observations were further confirmed at the molecular level by measuring specific chondrogenic marker Sox9, where
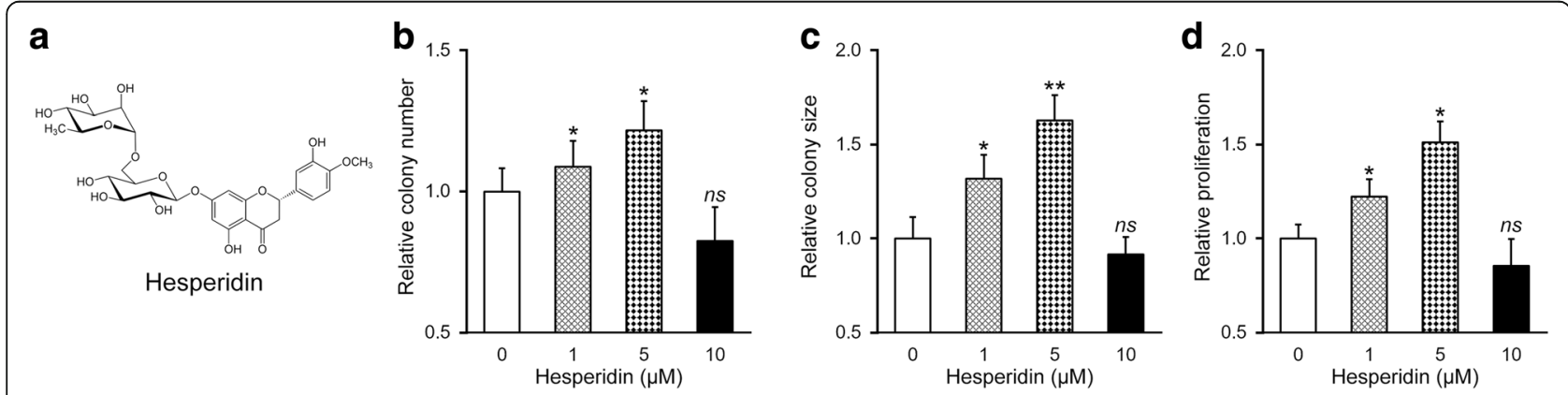

Fig. 1 Hesperidin improves self-renewal ability of MSCs. a Chemical structure of hesperidin. $\mathbf{b}$ to $\mathbf{d}$ Colony number (b), colony size (c) and proliferation (d) of MSCs after treatments with 0, 1, 5 and $10 \mu \mathrm{M}$ of hesperidin, respectively. Data were shown as mean \pm SD from at least three independent experiments. ${ }^{*} p<0.05,{ }^{* *} p<0.01$, ns not significant, versus $0 \mu \mathrm{M}$ hesperidin 


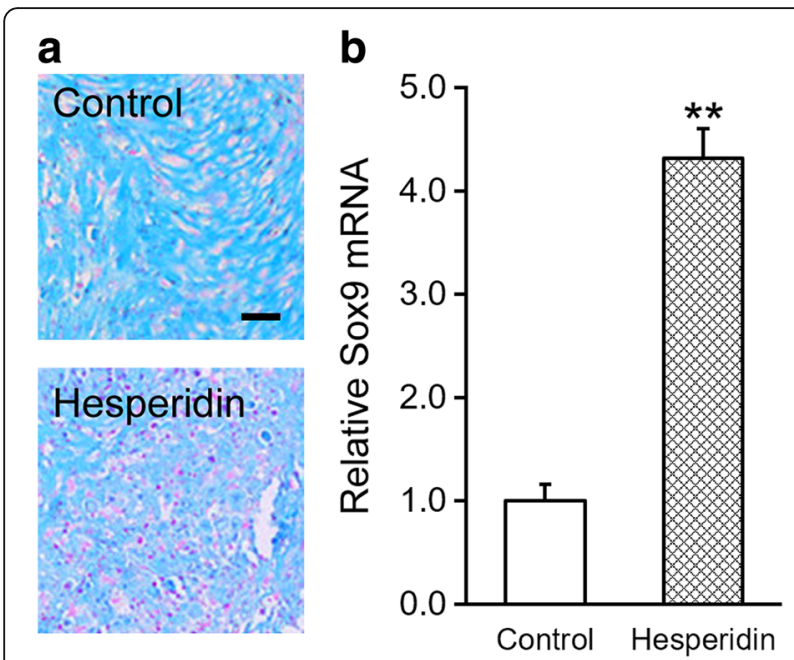

Fig. 2 Hesperidin enhances chondrogenesis of MSCs. a At day 14 after differentiation induction in the absence (control) or presence of $5 \mu \mathrm{M}$ hesperidin, the extents of chondrogenesis was evaluated by Alcian Blue staining assay. Images were representatives of at least three independent experiments, and positively stained cells were shown in pink, scale bar $100 \mu \mathrm{m}$. b At day 14 after differentiation induction in the absence (control) or presence of $5 \mu \mathrm{M}$ hesperidin, the extents of chondrogenesis was evaluated by mRNA levels of chondrogenic marker Sox9. Data were shown as mean \pm SD from at least three independent experiments. ${ }^{* *} p<0.01$, versus control

hesperidin treatment induced evident up-regulation of Sox-9 (Fig. 2b). Our results clearly demonstrated that, besides self-renewal ability, hesperidin also enhanced chondrogenesis of MSCs.

\section{Hesperidin suppresses secretion of pro-inflammatory cytokines}

Pro-inflammatory cytokines are essential players in both innate and acquired immune responses. Therefore, we subjected the MSCs in the absence or presence of $5 \mu \mathrm{M}$ hesperidin, and then measured the levels of pro-inflammatory cytokines IFN- $\gamma$, IL-2, IL-4 and IL-10 in the medium using ELISA. Results clearly indicated that hesperidin treatment inhibited the secretion of all of abovementioned cytokines compared with those of control (Fig. 3).

\section{Hesperidin inhibits the expression of nuclear factor kappa B (NF-KB) subunit p65}

To determine the extent of inflammation, we examined the expression of biomarkers in inflammatory responses, such as NF- $\mathrm{kB}$ subunit $\mathrm{p} 65$. We treated the MSCs in the absence or presence of $5 \mu \mathrm{M}$ hesperidin, and then examined the effect on expression of p65. We found that both mRNA and protein levels of p65 were significantly reduced by hesperidin treatment (Fig. $4 \mathrm{a}$ and b), indicating that hesperidin was able to inhibit the expression of NF- $\mathrm{B}$ subunit $\mathrm{p} 65$.

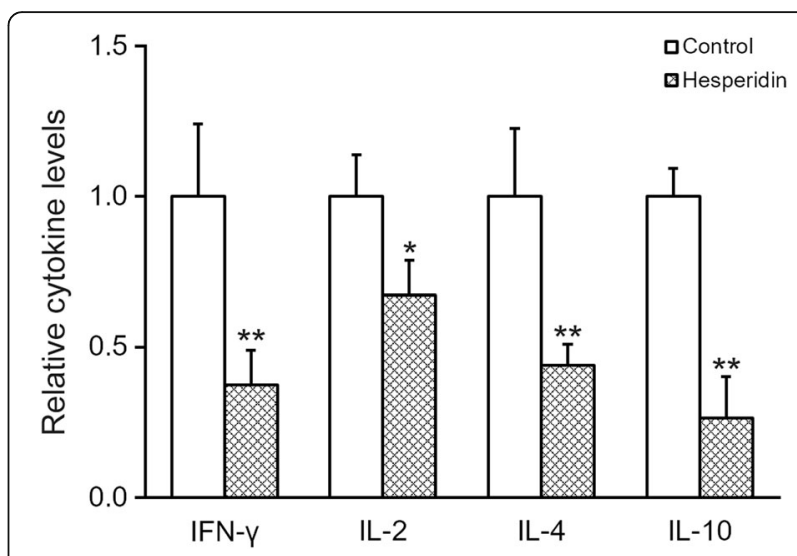

Fig. 3 Hesperidin inhibits secretion of IFN- $\gamma, I L-2, I L-4$ and IL-10 of MSCs. MSCs were treated in the absence (control) or presence of $5 \mu \mathrm{M}$ hesperidin, and levels of IFN- $\gamma, \mathrm{IL}-2, \mathrm{IL}-4$ and IL-10 in the medium were measured by ELISA. Data were shown as mean \pm SD from at least three independent experiments. ${ }^{*} p<0.05,{ }^{* *} p<0.01$, versus control

Next, to confirm the effect of hesperidin were due to decreased p65 expression, we introduced p65 siRNA knockdown in the MSCs (Additional file 1: Figure S1A and S1B). As expected, p65 knockdown significantly inhibited secretions of pro-inflammatory cytokines IFN- $\gamma$, IL-2, IL-4 and IL-10 (Additional file 1: Figure S1C). The MSCs were then subjected to differentiation for 14 days, after which stronger extent of chondrogenesis was observed in p65 knockdown cells, in terms of Sox9 mRNA expression (Additional file 1: Figure S1D) and Alcian Blue staining (Additional file 1: Figure S1E).

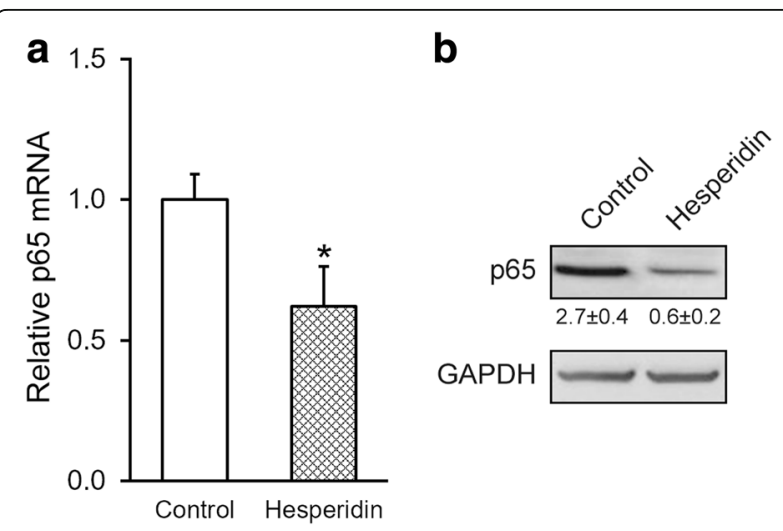

Fig. 4 Hesperidin suppresses the expression of nuclear factor kappa B (NF-KB) subunit p65 of MSCs. MSCs were treated in the absence (control) or presence of $5 \mu \mathrm{M}$ hesperidin, and relative mRNA (a) and protein (b) expressions of NF-KB subunit p65 were measured by RT$\mathrm{PCR}$ and Western blot, respectively. Western blot was representative of at least three independent experiments, with relative intensity $(\mathrm{p} 65 / \mathrm{GAPDH})$ indicated below as mean $\pm \mathrm{SD}$. Data were shown as mean \pm SD from at least three independent experiments. ${ }^{*} p<0.05$, versus control 
Inhibition of p65 is required for the inhibitory effect of hesperidin on cytokine secretions, and enhancing effect of hesperidin on chondrogenesis

We then questioned whether the inhibitory effect of hesperidin on p65 contributed to the earlier observed suppression on cytokine secretions. To this end, we overexpressed p65 in MSCs, and verified that both mRNA and protein levels of p65 were greatly elevated compared to baseline in the absence (control) or presence of $5 \mu \mathrm{M}$ hesperidin, respectively (Fig. 5a and b). Overexpression of p65 increased secretions of pro-inflammatory cytokines IFN- $\gamma$, IL-2, IL-4 and IL-10, which could be restored to baseline upon co-treatment of hesperidin (Fig. 6), suggesting that inhibition of p65 was indeed required for the inhibitory effect of hesperidin on cytokine secretions from MSCs.

Next, we further examined the effect of p65 inhibition on enhancing effect of hesperidin on chondrogenesis. MSCs were transduced with either empty control or p65 lentiviral particle, at day 14 after chondrogenesis induction in the absence (control) or presence of $5 \mu \mathrm{M}$ hesperidin. Overexpression of p65 decreased chondrogenesis in terms of Alcian Blue staining (Fig. 7a) and Sox9 expression (Fig. 7b). Again, upon co-treatment of hesperidin, chondrogenesis extent was restored to baseline, indicating that inhibition of p65 was also required for the enhancing effect of hesperidin on chondrogenesis of MSCs.

\section{Discussion}

In the study, we hereby reported for the first time that, hesperidin was able to improve self-renewal ability and chondrogenesis of MSCs, inhibit secretion of pro-inflammatory cytokines IFN- $\gamma$, IL-2, IL-4 and IL-10, and suppressed the expression of NF-kB subunit p65. Since human MSCs can be easily expanded and subsequently differentiated into matrix-producing chondrocytes [12, 13], eventually leading to the formation of hyaline articular cartilage, the observed enhancing effect of hesperidin on chondrogenesis of MSCs was potentially valuable in future clinical applications. In fact, hesperidin has been reported previously to exhibit beneficial effect toward cartilage tissues. In costal cartilage cells isolated from rabbits, a hesperidin loaded poly (lactic-co-glycolic acid) scaffold could improve attachment and proliferation of these cartilage cells, suggesting the potential of hesperidin in cartilage tissue engineering [22].

Furthermore, we overexpressed p65 in the context of hesperidin treatment, which reversed the hesperidin inhibited secretions of pro-inflammatory cytokines, and abolished the enhancing effect of hesperidin on chondrogenesis of MSCs. This result is intriguing in that it implicates the NF- $\kappa B$ signalling pathway and inflammation as the molecular mechanism underlying hesperidin action. NF- $\mathrm{kB}$, a nuclear transcription factor, is shown to be involved in inflammatory, immune and stress responses. The signalling pathway of NF-kB include NF- $k B$, NF- $k B$ inhibitor (ІкB), IKKs upstream kinase and I $\mathrm{B}$ kinase complex (IKKs). In mammals, NF- $\kappa B$ family is composed of NF-kB1, NF- $k$ B2, p65/RelA, C-Rel and RelB, all of which contain a Rel homology domain (RHD) [23]. NF- $\mathrm{kB}$ forms heterodimer or homodimer in cells, and p65/NF- $\mathrm{kB} 1$ is the first discovered and most widely existing dimer. The conventional NF- $\mathrm{kB}$ signaling also predominantly involves the $\mathrm{p} 65 / \mathrm{NF}-\kappa \mathrm{B} 1$ dimer [23]. NF- $\mathrm{KB}$ contributes to both innate and adaptive immune responses, and is one of the critical regulators of the production of pro-inflammatory cytokines [24]. The activation of NF- $\mathrm{kB}$ results in enhanced recruitment of inflammatory cells and increased production of proinflammatory mediators, including IL-1, IL-6, IL-8 and TNF. Inhibition of NF- $\mathrm{kB}$ activity has consistently

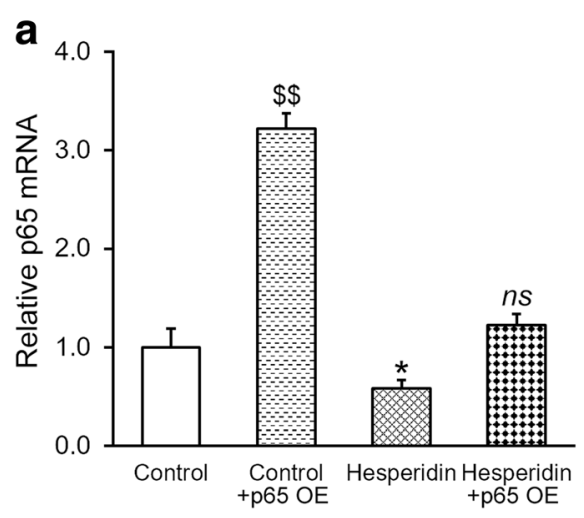

b

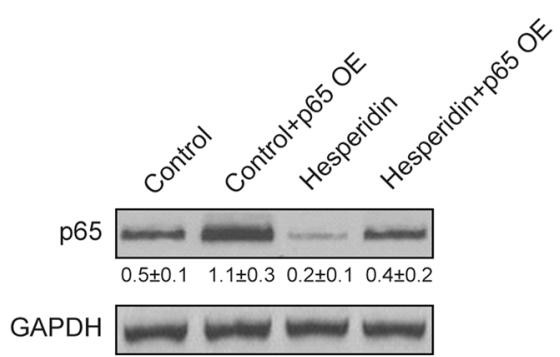

Fig. 5 Effects of p65 overexpression (OE) in MSCs. Relative mRNA (a) and protein (b) expressions of NF-kB subunit p65 were measured by RT-PCR and Western blot, respectively, after the MSCs were transduced with either empty control or p65 lentiviral particle, in the absence (control) or presence of $5 \mu \mathrm{M}$ hesperidin. Western blot was representative of at least three independent experiments, with relative intensity (p65/GAPDH) indicated below as mean \pm SD. Data were shown as mean \pm SD from at least three independent experiments. $\$ \$ p<0.01$, versus control, hesperidin and hesperidin+p65 OE. * $p<0.05$, versus control and hesperidin+p65 OE. ns not significant, versus control 


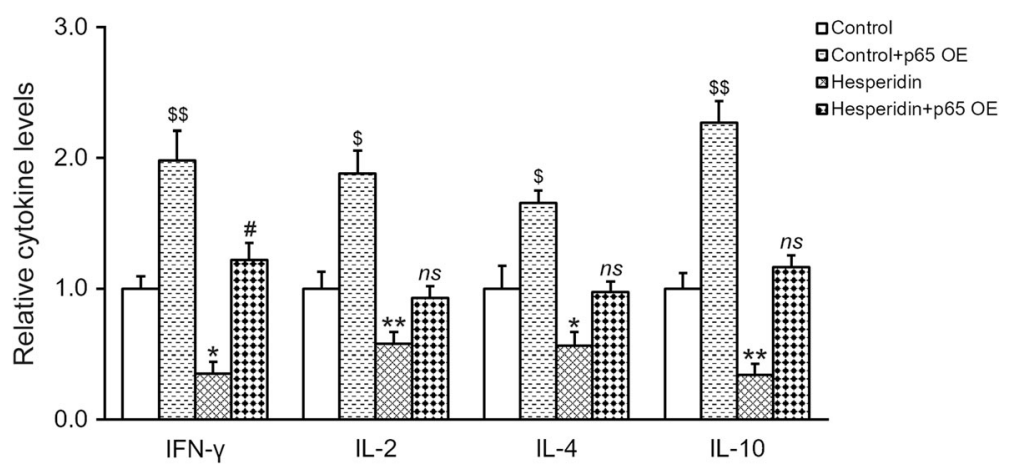

Fig. 6 Inhibition of p65 is required for the inhibitory effect of hesperidin on IFN- $\gamma$, IL-2, IL-4 and IL-10 secretions from MSCs. MSCs were transduced with either empty control or p65 overexpression (OE) lentiviral particle, in the absence (control) or presence of $5 \mu \mathrm{M}$ hesperidin, and levels of IFN- $\gamma$, IL-2, IL-4 and IL-10 in the medium were measured by ELISA. Data were shown as mean \pm SD from at least three independent experiments. \$ $\$ p<0.01, \$ p<0.05$, versus control, hesperidin and hesperidin+p65 OE. ${ }^{*} p<0.05,{ }^{*} p<0.01$, versus control and hesperidin+p65 OE. \# $p<0.05$, ns not significant, versus control

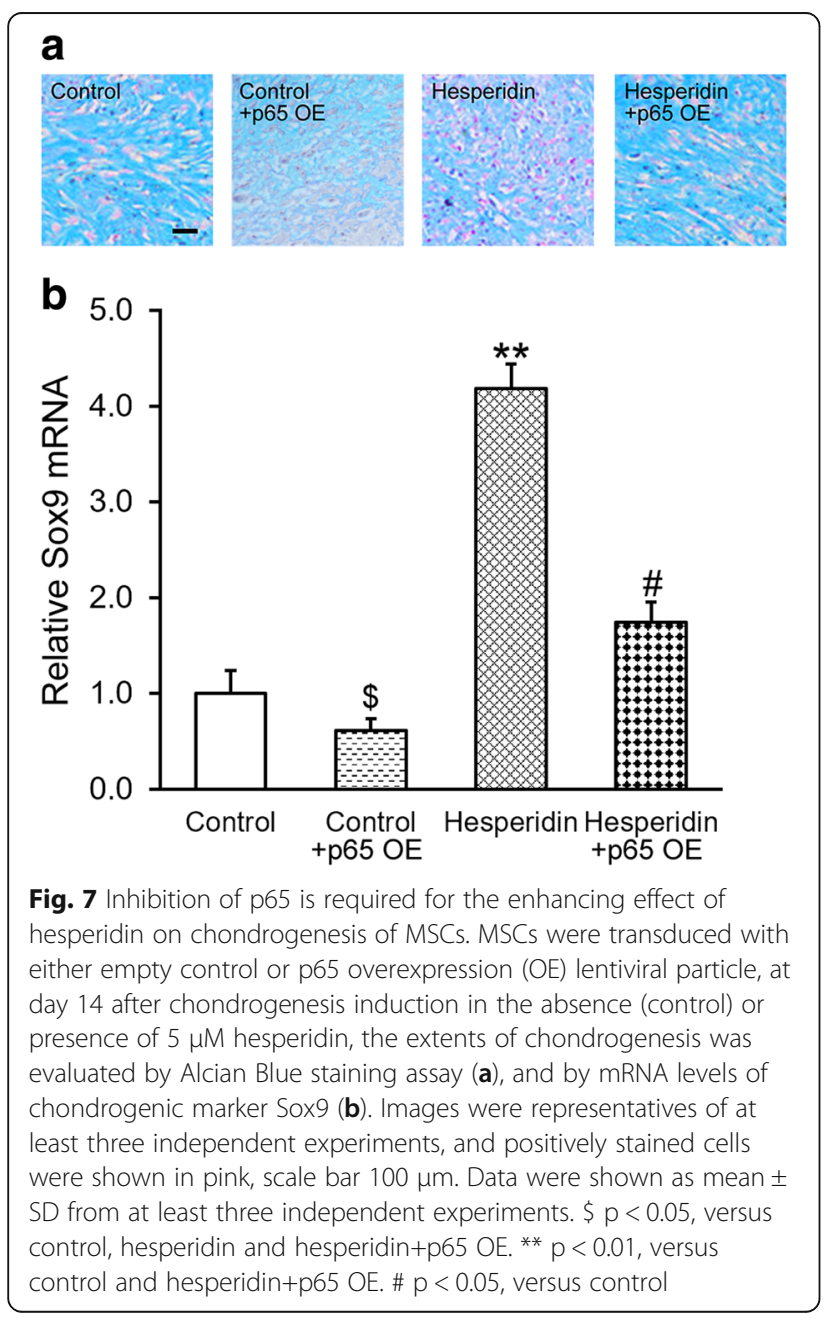

proven effective in the control of inflammatory diseases in several animal models. For example, blockage of NF- $\mathrm{kB}$ activity suppressed both the inflammation and tissue damage in rheumatoid synovium [25]. There has been reports regarding the effects of hesperidin on NF- $\mathrm{KB}$ signal transduction using mouse models. In a diabetic mouse model, hesperidin reduced NF- $\mathrm{B}$ level [26], whereas in a mouse model for pain hesperidin was shown to suppress the activity of NF-kB [19]. Further, hesperidin was also demonstrated to negatively regulate pro-inflammatory cytokines downstream of NF- $\mathrm{BB}$, including IL-6 and IL-10 [21]. Our current study provides yet another instance supporting the anti-inflammatory properties of hesperidin, indicating that hesperidin may possess a universal anti-inflammatory function in various disease models, including but not limited to cartilage repair via tissue engineering using MSCs.

It is challenging to produce tissue-engineered cartilage resembling the native articular cartilage. Mechanical loading is regarded as a critical factor in cartilage tissue engineering for the reason that in daily activities normal articular cartilage is constantly subjected to mechanical loading [6, 27]. Mechanical loading is able to stimulate chondrogenesis in vitro and suppress hypertrophic differentiation of human MSCs [28, 29]. However, the particular type of mechanical loading and its loading regime to enhance non-hypertrophic chondrogenesis along with the mechano-transduction signalling remain to be clarified. This knowledge will help to establish a function-wise native-like tissue-engineered articular cartilage for therapeutic applications [27], and it would be of great clinical value to investigate whether hesperidin treatment could enhance the mechanical response of cartilage tissue derived from human MSCs.

Other factors contributing to successful cartilage tissue engineering using human MSCs include three-dimensional (3D) scaffold, and growth factors, both of which are 
reported to be essential for the quality of tissue-engineered cartilage $[2,10,30]$. In the context, the study by Cho et al. on the effect of hesperidin loaded poly (lactic-co-glycolic acid) scaffold costal cartilage cells [22] has demonstrated the usefulness of combining 3D scaffold with hesperidin. It will be interesting to further investigate the combinational effect of hesperidin with other contributing factors on MSC chondrogenesis in future studies.

\section{Conclusion}

To conclusion, our current study demonstrates that hesperidin serves as a therapeutic agent to effectively enhance both self-renewal and chondrogenesis of human MSCs in vitro by inhibiting inflammation to facilitate cartilage tissue repair. Our current study therefore supports a wider application of hesperidin for multiple approaches to use MSCs for clinical cartilage tissue repair.

\section{Methods}

\section{Human MSC culturing}

The protocol for the use of human cells was approved by the committee of the Second Hospital of Shandong University, and bone marrow cells were harvested from bone fragments of patients from the Second Hospital of Shandong University, with written consent forms acquired from all patients. All bone marrow aspirates were diluted with low-glucose Dulbecco's modified Eagle's medium (DMEM, Thermo, Waltham, MA, USA), and then subjected to Ficoll gradient centrifugation $(1200 \times \mathrm{g}$ for $30 \mathrm{~min}$ at room temperature). The cells from the interface were harvested, followed by two washes in phosphate-buffered saline (PBS). Mononuclear cells re-suspended in complete DMEM were counted with a hemocytometer and seeded in $10 \mathrm{~cm}^{2}$ tissue culture dishes at a density of $5 \times 10^{6}$ cells $/ 10 \mathrm{~mL}$. After two days, floating cells were discarded, and the adherent cells were kept for culture at $37{ }^{\circ} \mathrm{C}$ with $5 \%$ humidified $\mathrm{CO}_{2}$. After reaching a confluence of $75-85 \%$, cells were detached with $0.05 \%$ trypsin/1 mM EDTA and re-plated, and expanded cells with $<9$ passages were used in the experiments.

\section{Colony formation assay}

A total of $1 \times 10^{5} \mathrm{MSCs}$ were plated into a 10 -cm petri dish and continuously cultured for up to 21 days in the presence of $0,1,5$ and $10 \mu \mathrm{M}$ of hesperidin, respectively. Crystal violet $(0.5 \%$, SIGMA, MO, USA) was used to stain the formed colonies for 15 mins followed by counting under light microscope. Colonies larger than $2 \mathrm{~mm}$ in diameter were counted, which typically ranged from 50 to 200 per dish (calculated from 10 randomly chosen fields in each dish).

\section{Proliferation assay}

The proliferation of cells was assessed by commercial CCK-8 kit (Dojindo, Kumamoto, Japan). In brief, $1 \times 10^{5}$ MSCs were plated into each well of 6-well plate and continuously cultured for up to 7 days in the presence of $0,1,5$ and $10 \mu \mathrm{M}$ of hesperidin, respectively. $10 \mathrm{ul}$ CCK-8 solution was then added into each well and the chromogenic reaction was carried out at $37{ }^{\circ} \mathrm{C}$ for 15 mins. Microplate reader (Molecular Devices, Sunnyvale, CA, USA) was used to record the absorption at $450 \mathrm{~nm}$ and relative cell viability was calculated.

\section{Chondrogenesis assays}

MSCs were cultured in chondrogenic induction medium (DMEM, $0.2 \mathrm{mM}$ ascorbate-2-phosphate, 20\% FBS, and $10 \mathrm{mM}$ glycerol-2-phosphate) for 14 days in the absence or presence of $5 \mu \mathrm{M}$ hesperidin, with fresh medium exchanged every 2 days. Chondrogenesis was evaluated using Alcian Blue (Millipore, Billerica, MA, USA) staining.

\section{mRNA extraction and real-time PCR}

Total mRNA was extracted using Trizol (Invitrogen, Carlsbad, CA, USA), and reverse transcribed to complementary cDNAs with Superscript II following manufacturer's instructions (Biorad, Hercules, CA, USA). Triplicate PCR reactions were conducted using cyber green-based system (Applied Biosystems, Waltham, MA, USA) with the following conditions: $15 \mathrm{~s}$ at $95^{\circ} \mathrm{C}, 1 \mathrm{~min}$ at $60{ }^{\circ} \mathrm{C}$ for 40 times. The relative expression levels were calculated using GAPDH as the internal control. Primers used in this study were: Sox9 forward 5'-GTA CCC GCA CTT GCA CAA-3', reverse 5'-TCT CGC TCT CGT TCA GAA GTC-3'; p65 forward 5'-ACA TCC ATG CGG AGA ACG AGG AG-3', reverse 5'-AGT GCT GCG AGT GAG TCA AGA GG-3'; GAPDH forward 5'-CTG ACT TCA ACA GCG ACA CC-3', reverse 5'-TAG CCA AAT TCG TTG TCA TAC-3'.

\section{Western blot}

Cell resuspension was prepared in the lysis buffer containing $150 \mathrm{mM} \mathrm{NaCl}, 50 \mathrm{mM}$ Tris- $\mathrm{HCl}, 10 \mathrm{mM}$ HEPES, $0.1 \%$ NP-40 alternative, $0.5 \mathrm{mM} \mathrm{NaF,} 0.25 \%$ Na-deoxycholate, $1 \mathrm{mM} \mathrm{Na} \mathrm{VO}_{4}$, pH 7.4 (Protease Inhibitor Cocktail, Roche, 1 tablet/10 ml). Cell lysates were quantitated using BCA protein assays, and $30 \mu \mathrm{g}$ total protein was then run on SDS-PAGE followed by transfer to PVDF membranes. The membranes were subsequently blocked with $1 \%$ BSA (bovine serum albumin, Sigma, USA), and incubated with primary antibodies at $4{ }^{\circ} \mathrm{C}$ overnight. Primary antibodies for p65 and GAPDH were both purchased from Abcam. HRP conjugated secondary antibodies were utilized to visualize bands in an ECL-based imaging system. 


\section{p65 overexpression and knockdown}

Stable overexpression and knockdown of p65 were established using p65 lentiviral particle (LPP-F0160-Lv105) and p65 shRNA particle (HSH016213-CH1), both of which purchased from GeneCopoeia (Rockville, MD, USA). Cells were first transduced by respective lentiviral particles for $24 \mathrm{~h}$, followed by selection with puromycin for 2 weeks, according to vendor's instructions.

\section{Enzyme-linked immunosorbent assay (ELISA)}

The MSCs were treated in the absence or presence of $5 \mu \mathrm{M}$ hesperidin for 2 days. Cells were then completely removed by centrifugation and clear medium was collected for ELISA analysis. The levels of IFN- $\gamma$, IL-2, IL-4 and IL-10 were measured with the commercially available ELISA kits (Abcam, MA, USA) following the manufacturer's instructions.

\section{Statistical analysis}

All data were analyzed using SPSS 22.0 system (IBM, Armonk, NY, USA), and presented as mean \pm standard deviation (SD) from at least three independent experiments. The differences between groups were determined by Student's $T$ tests and single factor variance analysis (ANOVA). $P$ values less than 0.05 were considered statistical significant.

\section{Additional file}

Additional file 1: Figure S1. p65 knockdown inhibits secretion of IFN- $\gamma$ IL-2, IL-4 and IL-10, and enhances chondrogenesis of MSCs. MSCs were transduced with control or siRNA against NF-KB subunit p65, followed by assessments of (A) mRNA and (B) protein expressions of p65, (C) levels of IFN- $\gamma, I L-2, I L-4$ and IL-10 in the medium. MSCs with either control or p65 siRNA were subjected to 14 days of differentiation induction, followed by assessments of (D) mRNA expression of chondrogenic marker Sox9, and (E) extents of chondrogenesis. Images were representatives of at least three independent experiments, and positively stained cells were shown in pink, scale bar $100 \mu \mathrm{m}$. Western blot was representative of at least three independent experiments, with relative intensity (p65/GAPDH) indicated below as mean $\pm S D$. Data were shown as mean \pm SD from at least three independent experiments. ${ }^{* *} p<0.01,{ }^{*} p<0.05$, versus control. (DOCX $519 \mathrm{~kb})$

\section{Abbreviations}

MSCs: Mesenchymal stem cells; NF-kB: Nuclear factor kappa B

\section{Acknowledgements}

Not applicable.

\section{Funding}

This work was supported by the Youth Fund of the Second Hospital of Shandong University (Y2014010028) and the Key Research And Development Plan of Shandong Provence (No. 2015GSF118107).

\section{Availability of data and materials}

All data generated or analysed during this study are included in this published article.

\section{Authors' contributions}

$S X, W L, J B, S L, H Z, N G, D X, H G$ performed the experiments, analyzed and interpreted the data. MG designed the study and wrote the manuscript. All authors read and approved the final manuscript.

\section{Ethics approval and consent to participate}

This study was approved by the ethics committee of The Second Hospital of Shandong University, and followed the ethical guidelines laid down in the 1975 Declaration of Helsinki. All participants have given informed and written consent.

\section{Consent for publication}

All participants have given consent for publication.

\section{Competing interests}

The authors declare that they have no competing interests.

\section{Publisher's Note}

Springer Nature remains neutral with regard to jurisdictional claims in published maps and institutional affiliations.

\section{Author details}

${ }^{1}$ Department of Joint Surgery, The Second Hospital of Shandong University, Jinan 250033, Shandong, China. ${ }^{2}$ Key Laboratory for Liquid-Solid Structural Evolution and Processing of Materials, Ministry of Education, Shandong University, Jinan 250061, Shandong, China. ${ }^{3}$ Department of emergency, The Second Hospital of Shandong University, Jinan 250033, Shandong, China. ${ }^{4}$ Department of Trauma and Orthopedics, The Second Hospital of Shandong University, Jinan 250033, Shandong, China

Received: 8 April 2018 Accepted: 13 July 2018

Published online: 20 July 2018

References

1. Madeira C, Santhagunam A, Salgueiro JB, Cabral JM. Advanced cell therapies for articular cartilage regeneration. Trends Biotechnol. 2015;33:35-42.

2. Vega SL, Kwon MY, Burdick JA. Recent advances in hydrogels for cartilage tissue engineering. Eur Cell Mater. 2017;33:59-75.

3. Bernhard JC, Vunjak-Novakovic G. Should we use cells, biomaterials, or tissue engineering for cartilage regeneration? Stem Cell Res Ther. 2016;7:56.

4. Ofek G, Dowling EP, Raphael RM, McGarry JP, Athanasiou KA. Biomechanics of single chondrocytes under direct shear. Biomech Model Mechanobiol. 2010;9:153-62.

5. Ahmed TA, Hincke MT. Strategies for articular cartilage lesion repair and functional restoration. Tissue Eng Part B Rev. 2010;16:305-29.

6. Tan AR, Hung CT. Concise review: mesenchymal stem cells for functional cartilage tissue engineering: taking cues from chondrocyte-based constructs. Stem Cells Transl Med. 2017;6:1295-303.

7. Centers for Disease. C, prevention: prevalence of doctor-diagnosed arthritis and arthritis-attributable activity limitation--United States, 2010-2012. MMWR Morb Mortal Wkly Rep. 2013;62:869-73.

8. Lawrence RC, Felson DT, Helmick CG, Arnold LM, Choi H, Deyo RA, Gabriel S, Hirsch R, Hochberg MC, Hunder GG, et al. Estimates of the prevalence of arthritis and other rheumatic conditions in the United States. Part II. Arthritis Rheum. 2008;58:26-35.

9. Gaut C, Sugaya K. Critical review on the physical and mechanical factors involved in tissue engineering of cartilage. Regen Med. 2015;10:665-79.

10. Bornes TD, Adesida AB, Jomha NM. Mesenchymal stem cells in the treatment of traumatic articular cartilage defects: a comprehensive review. Arthritis Res Ther. 2014;16:432.

11. Yamasaki S, Mera H, Itokazu M, Hashimoto Y, Wakitani S. Cartilage repair with autologous bone marrow mesenchymal stem cell transplantation: review of preclinical and clinical studies. Cartilage. 2014;5:196-202.

12. Yong KW, Wan Safwani WK, Xu F, Wan Abas WA, Choi JR, PingguanMurphy B. Cryopreservation of human mesenchymal stem cells for clinical applications: current methods and challenges. Biopreserv Biobank. 2015;13:231-9.

13. Yong KW, Choi JR, Wan Safwani WK. Biobanking of human mesenchymal stem cells: future strategy to facilitate clinical applications. Adv Exp Med Biol. 2016;951:99-110. 
14. Saito T, Yano F, Mori D, Kawata M, Hoshi K, Takato T, Masaki H, Otsu M, Eto K, Nakauchi $\mathrm{H}$, et al. Hyaline cartilage formation and tumorigenesis of implanted tissues derived from human induced pluripotent stem cells. Biomed Res. 2015; 36:179-86.

15. Chung C, Burdick JA. Engineering cartilage tissue. Adv Drug Deliv Rev. 2008;60:243-62.

16. Klein TJ, Malda J, Sah RL, Hutmacher DW. Tissue engineering of articular cartilage with biomimetic zones. Tissue Eng Part B Rev. 2009;15:143-57.

17. Makris EA, Gomoll AH, Malizos KN, Hu JC, Athanasiou KA. Repair and tissue engineering techniques for articular cartilage. Nat Rev Rheumatol. 2015:11:21-34.

18. Zhang W, Moskowitz RW, Nuki G, Abramson S, Altman RD, Arden N, BiermaZeinstra S, Brandt KD, Croft P, Doherty M, et al. OARSI recommendations for the management of hip and knee osteoarthritis, part II: OARSI evidencebased, expert consensus quidelines. Osteoarthritis Cartilage. 2008;16:137-62.

19. Pinho-Ribeiro FA, Hohmann MS, Borghi SM, Zarpelon AC, Guazelli CF, Manchope MF, Casagrande R, Verri WA Jr. Protective effects of the flavonoid hesperidin methyl chalcone in inflammation and pain in mice: role of TRPV1, oxidative stress, cytokines and NF-kappaB. Chem Biol Interact. 2015; 228:88-99.

20. Martinez RM, Pinho-Ribeiro FA, Steffen VS, Caviglione CV, Vignoli JA, Baracat MM, Georgetti SR, Verri WA Jr, Casagrande R. Hesperidin methyl chalcone inhibits oxidative stress and inflammation in a mouse model of ultraviolet $B$ irradiation-induced skin damage. J Photochem Photobiol B. 2015;148:145-53.

21. Martinez RM, Pinho-Ribeiro FA, Steffen VS, Caviglione CV, Pala D, Baracat MM, Georgetti SR, Verri WA, Casagrande R. Topical formulation containing hesperidin methyl chalcone inhibits skin oxidative stress and inflammation induced by ultraviolet B irradiation. Photochem Photobiol Sci. 2016;15:554-63.

22. Cho SA, Cha SR, Park SM, Kim KH, Lee HG, Kim EY, Lee D, Khang G. Effects of hesperidin loaded poly(lactic-co-glycolic acid) scaffolds on growth behavior of costal cartilage cells in vitro and in vivo. J Biomater Sci Polym Ed. 2014;25:625-40.

23. Wang R, Zhang L, Zhang X, Moreno J, Celluzzi C, Tondravi M, Shi Y. Regulation of activation-induced receptor activator of NF-kappaB ligand (RANKL) expression in T cells. Eur J Immunol. 2002;32:1090-8.

24. Li Q, Verma IM. NF-kappaB regulation in the immune system. Nat Rev Immunol. 2002;2:725-34.

25. Bondeson J, Foxwell B, Brennan F, Feldmann M. Defining therapeutic targets by using adenovirus: blocking NF-kappaB inhibits both inflammatory and destructive mechanisms in rheumatoid synovium but spares antiinflammatory mediators. Proc Natl Acad Sci U S A. 1999;96:5668-73.

26. Iskender H, Dokumacioglu E, Sen TM, Ince I, Kanbay Y, Saral S. The effect of hesperidin and quercetin on oxidative stress, NF-kappaB and SIRT1 levels in a STZ-induced experimental diabetes model. Biomed Pharmacother. 2017; 90:500-8.

27. Shieh AC, Athanasiou KA. Principles of cell mechanics for cartilage tissue engineering. Ann Biomed Eng. 2003;31:1-11.

28. Schatti O, Grad S, Goldhahn J, Salzmann G, Li Z, Alini M, Stoddart MJ. A combination of shear and dynamic compression leads to mechanically induced chondrogenesis of human mesenchymal stem cells. Eur Cell Mater. 2011;22:214-25.

29. Zhang T, Wen F, Wu Y, Goh GS, Ge Z, Tan LP, Hui JH, Yang Z. Cross-talk between TGF-beta/SMAD and integrin signaling pathways in regulating hypertrophy of mesenchymal stem cell chondrogenesis under deferral dynamic compression. Biomaterials. 2015;38:72-85.

30. Ringe J, Burmester GR, Sittinger M. Regenerative medicine in rheumatic disease-progress in tissue engineering. Nat Rev Rheumatol. 2012;8:493-8.

Ready to submit your research? Choose BMC and benefit from:
- fast, convenient online submission
- thorough peer review by experienced researchers in your field
- rapid publication on acceptance
- support for research data, including large and complex data types
- gold Open Access which fosters wider collaboration and increased citations
- maximum visibility for your research: over 100M website views per year
At BMC, research is always in progress.
Learn more biomedcentral.com/submissions

